\title{
Accuracy of BLUP breeding values in a factorial mating design with mixed families and marker-based parentage assignment in rainbow trout Oncorhynchus mykiss
}

\author{
Haffray Pierrick ${ }^{1,{ }^{*}}$, Enez Florian ${ }^{1}$, Bugeon Jérôme ${ }^{2}$, Chapuis Hervé ${ }^{3}$, Dupont-Nivet Mathilde ${ }^{4}$, \\ Chatain Beatrice ${ }^{5}$, Vandeputte Marc ${ }^{4,5}$
}

${ }^{1}$ SYSAAF, LPGP, Campus de Beaulieu, F-35042 Rennes, France

2 INRA, UR1037, LPGP, Rennes, F-35042, France

${ }^{3}$ SYSAAF, SRA/INRA, F-37380 Nouzilly, France

${ }^{4} \mathrm{GABI}$, INRA, AgroParisTech, Université Paris-Saclay, F-78350 Jouy-en-Josas, France

${ }^{5}$ Ifremer, Chemin de Maguelone, F-34250 Palavas-les-Flots, France

* Corresponding author : Pierrick Haffray, email address : pierrick.haffray@inra.fr

\begin{abstract}
:
Marker-based parentage assignment provides the opportunity to investigate factors of efficiency for mixed-family designs and factorial mating. In such designs, family size is both uncontrolled and small, which may be thought to limit the accuracy of estimated breeding values (EBVs). The objective of this work was to estimate the accuracy of EBVs of growth and quality traits in a large factorial mating design and in commercial breeding conditions.
\end{abstract}

An expected six hundred full-sib families of rainbow trout Oncorhynchus mykiss (2042 fish in total) were produced by ten factorial matings of six dams with ten sires. Fish were phenotyped for body weight, carcass yield, fillet yield, fillet fat content and fillet colour, and family information was recovered using microsatellite markers.

The accuracy of EBVs was estimated using or removing individual performance to mimic combined family selection (with individual phenotype) or sib selection (without individual phenotype).

The traits investigated had medium to high heritability $(0.17-0.58)$. High to very high accuracy $(0.630-$ 0.817 ) was estimated for combined family selection. The accuracy of sib selection (not using individual phenotype) was 18-22\% lower (0.542-0.638), but remained in the upper range reported for such traits.

This level of accuracy was higher than those reported in conventional breeding programs using separate family rearing. This was true even for families with a very low number of full-sibs. Individual EBV accuracy was more closely linked to the total number of full- and half-sibs of each fish than to its number of full-sibs. We hypothesize that this was due to the factorial mating, which led to a high number of the genetic ties between sibs. 
These results highlight the possibility of introducing precise estimated breeding values for quality traits into combined or sib selection in breeding programs when using mixed families from factorial designs and marker-based parentage assignment in aquaculture species.

\section{Highlights}

- This work reports for the first time accuracies of EBV in a mixed family breeding design assisted by DNA-parentage assignment for growth and quality traits (0.6-0.8). Theses accuracies were higher than those reported in classical family-based breeding program and similar or higher than reported by simulation for genomic selection - The factorial mating is proposed as the factor that allow such interesting advantage - This result confirms the potential interest to use such design to initiate domestication and selective breeding program

Keywords : Aquaculture, Salmonids, DNA parentage assignment, Sib selection, EBV accuracy 


\section{Introduction}

Less than $10 \%$ of world aquaculture production benefits from genetically improved seed. When it does, this comes mostly from conventional family-based breeding programs (Gjedrem et al., 2012), using families reared separately until tagging and Best Linear Unbiased Prediction, or BLUP (Henderson, 1984). One of the major reasons for this limited development is the high cost of these programs, which require both initial investment in numerous family tanks $(>100)$ and highly trained staff using very good traceability and rearing practices to limit both pedigree errors and initial tank effect. This is a serious obstacle for small and medium enterprises (SMEs) and for the initiation of selective breeding in new aquaculture species.

Alternative breeding schemes using marker-based parentage assignment and combining mass selection on traits measurable in candidates (such as growth) and sib selection on lethal traits (quality, disease resistance, etc.) have not been greatly studied so far (Vandeputte and Haffray, 2014). This kind of breeding scheme limits both tank effect and initial investment, as all families can be reared together in just one tank. The use of common garden rearing and molecular pedigrees also relieves constraints on family number, allowing the production of more families for a fixed number of parents (e.g., by the use of factorial mating designs). This increases the potential for between-family selection intensity at a given level of inbreeding (Dupont-Nivet et al., 2006; Sorensen et al., 2005).

The relative efficiency of breeding programs can be assessed by the accuracy $\left(r_{A \hat{A}}\right)$ of the estimated breeding values (EBVs). This accuracy is the correlation between the true breeding value (TBV) and the EBV, and can be derived from mixed-model equations (Van Vleck, 1993).

In aquaculture, there are few reports on the accuracy of EBVs for production traits, with only two references available on conventional breeding schemes with initially separated families 
(Gonzalez-Pena et al., 2016; Kause et al., 2007) and one on a mixed families scheme (Blonk et al., 2010). One problem with the mixed-families design, using a posteriori parentage assignment, is that the number of sibs per family is variable and unpredictable. In addition, as factorial designs generate very high numbers of families, they can result in very low numbers of fish recorded from each family. Small and variable family sizes could be seen as unfavourable to the accuracy of breeding values.

The aim of the present work was to estimate the accuracy of EBVs for different traits (growth, carcass traits and fillet quality) in a factorial mating design in rainbow trout Oncorhynchus mykiss using a posteriori parentage assignment. Genetic parameters, EBVs and $r_{A \hat{A}}$ were estimated within a single generation using performances collected from a random sample of 2000 offspring. Both univariate and multivariate models were investigated as the literature mostly reports evaluations or simulations with univariate models, but breeding programs generally rely on multivariate models. The accuracy of sib selection was compared with combined family selection for each trait and with univariate or multivariate models using a leave-one-out (LOO) procedure, where the phenotype of any given individual was either used (combined selection) or not (sib selection).

These estimates of accuracy in a mixed-family design in aquaculture are expected to support the introduction of combined family or sib-based selection on quality traits in mass selection programs initially designed to improve growth.

\section{Methods}

2.1. Management and phenotyping of animals

The measured traits and rearing environments were indexed according to the ATOL (Animal Trait Ontology for Livestock) and EOL (Environment Ontology for Livestock) ontologies 
available on the ATOL (http://www.atol-ontology.com/index.php/en/les-ontologiesen/visualisation-en) website.

The offspring sample had already been used to estimate genetic parameters and design predictors for quality traits in previous studies (Haffray et al., 2012a; Haffray et al., 2013) and was established from a commercial line (Aqualande breeding company, Pissos, France). Six hundred all-female full-sib families from 60 dams and 100 sires were produced in one day (November 2004) through a partial factorial mating design with 10 independent blocks. Each block was the full factorial mating of 6 dams with 10 sex-reversed sires (neomales) and thus comprised 60 families. A piece of fin from each parent was kept in $95 \%$ ethanol for later marker-based parentage assignment. Eggs from maternal half-sib families were incubated separately by dam. At eyed stage, non-genetic maternal effects were managed by creating 12 new batches, each grouping spawns from 5 dams with similar mean egg size (Haffray et al., 2012b). Each batch was then expected to contain $5 \times 10=50$ distinct full-sib families. Each batch was reared separately until 147 days post fertilization (D147) when they had reached the same mean length (BL, ATOL: 0001658) of $9.7 \pm 0.7 \mathrm{~cm}$. At this age, and before any selection, 250 individuals per batch $(\mathrm{n}=3000$ in total) were sampled at random and pooled (i.e., from 600 expected families). At D198 (BW = $73.2 \pm 17.6 \mathrm{~g}$ ), individuals were individually tagged with RFID transponders (Biolog-ID, France) and a piece of fin was preserved in $95 \%$ ethanol. At D220, fish were transferred to the "Viviers de la Hountine" fish farm (Belin-Béliet, France).

Water temperature varied from 3 to $20^{\circ} \mathrm{C}$ during the year. Tanks and raceways were supplied with the "first" water just entering in the fish farm. Fish were vaccinated against Yersinia ruckeri at D220. They were grown under non limiting oxygen availability (>80\% oxygen saturation, EOL:0000186) and fed to satiation using Neo Extra extruded commercial feed (Le Gouessant, Lamballe, France; $43 \%$ protein and 23\% lipids) from $40 \mathrm{~g}$ to $300 \mathrm{~g}$ and Neo Ultra 
(41\% protein and $26 \%$ lipids; $25 \mathrm{ppm}$ astaxanthine) from $300 \mathrm{~g}$ to the final harvesting. Density (EOL: 0000043) increased with growth, but was kept to a maximum of $70 \mathrm{~kg} / \mathrm{m}^{3}$.

For final phenotyping, fish were divided at random into two equal subgroups and slaughtered on two different dates (D509 and D511) in order to reduce the waiting time between slaughtering and phenotyping. Each sub-group was fasted for 3 days, anaesthetised $\left(\mathrm{CO}_{2}\right)$, killed by bleeding in icy water at the Aqualande processing plant (Roquefort, France, $50 \mathrm{~km}$ from the fish farms) and transported in a refrigerated truck to the IFREMER experimental facility (Nantes, France) for processing and data collection.

\subsection{Data collection and parentage assignment}

The fish were phenotyped for body weight (BW in g, ATOL: 0000351), gutted carcass yield (Car\%, ATOL: 0000548), fillet yield (Fil\%, ATOL: 0002305) as previously reported (Haffray et al., 2012a), as well as fillet fat content (Fat\%; ATOL: 0001663) and redness (a*; ATOL: 0001017). Fat\% was indirectly estimated by microwave absorption (Distell Fish Fat Meter) as the mean of the Fat Meter measurements at the anterior and the posterior dorsal positions above the lateral line of the left side of the fish (Douirin et al., 1998). Redness of the fillet was measured according to the international CIE L*a*b* colour space model (CIE, 1976) using a Minolta CR200 Chroma Meter to obtain the mean value of the $\mathrm{a}^{*}$ component at three dorsal positions on the left fillet (anterior, medium or posterior). Malformed fish (ATOL: 0002265) were identified by visual observation of the vertebral axis after filleting. The very few fish found to have malformations of the vertebral axis $(0.07 \%)$ were discarded from further analysis.

Fish were assigned to their parents by a private laboratory for livestock genotyping (Labogena, ISO 17025 accredited, Jouy en Josas, France) using thirteen microsatellites. Only fish unambiguously assigned by exclusion to both their parents with a maximum of two 
authorized mismatches were kept for statistical analysis. As previously reported (Haffray et al., 2012b), parthenogenetic autotriploids $(n=29)$ were discarded from the statistical analysis.

\subsection{Genetic parameters}

The weight of the carcass and fillets, as well as muscle fat $\%$ and $\mathrm{a}^{*}$ are phenotypically linked to body weight. As commercial body weight was not expected to increase following selection (i.e., slaughter weight is fixed by the market), these traits were regressed on BW and the residual of the regression was used as the phenotype. For carcass weight and fillet weight, phenotypes at a fixed mean body weight were used as a proxy for the ratio (carcass yield Carc\% and fillet yield Fil\%, respectively) as suggested by Koch et al. (1963) to avoid spurious genetic correlations between the ratio and its denominator, in this case body weight.

Day of slaughter (2 levels), number of days after slaughtering (3 levels) and filleting operator (2 levels) were significant fixed effects (PROC MIXED, SAS; $p<0.01$ ) for Fil\% and the first two of these effects were significant for fillet a* colour.

Significance of maternal effect was tested (Chi-2 test) by comparing log-likelihoods (-2LL) of models with or without maternal effect (Haffray et al., 2012b), and was never significant.

Heritabilities $\left(\mathrm{h}^{2}\right)$ and EBVs were estimated with REMLF90 (Misztal et al., 2002) with an animal model and fixed effects when significant (see above). Data were normalized with the following formula, $x_{\text {new }}=[\mathrm{x}-\mu] / \sigma$, where $\mathrm{x}$ is the phenotypic performance measured, $\mu$ is its mean and $\sigma$ is its phenotypic standard deviation; so that all transformed traits had zero mean and unity variance. Univariate models (to enable comparison with previously published results) and multivariate models were applied to estimate accuracy. Multivariate models included all of the following traits: BW, Carc\%, Fil\%, Fat $\%$ and a*.

\subsection{Accuracy of EBVs}


The accuracy of EBVs $\left(r_{A \hat{A}}\right)$ was calculated as the square root of the $r_{A \hat{A}}^{2}$ determination coefficient (or CD) equal to $1-[\operatorname{var}(\hat{u}-u) / \operatorname{var} u]$, where $\hat{u}$ is the trait $\mathrm{EBV}, u$ the true but unknown BV and var $u$ the estimated genetic variance of the trait. For each individual, var $(\hat{u}$ $-u$ ), also called prediction error variance $(\mathrm{PEV})$, was calculated as the square of the standard error (SE) of each EBV with the above-mentioned univariate or multivariate models. These accuracies corresponded to a combined family-based breeding program with performance also measured on candidates. In order to evaluate the accuracy of sib selection (with no performance measured on the candidates), the EBVs of each individual were also alternatively estimated without using their own performance in the model, in a leave-one-out (LOO) crossvalidation procedure (Arlot and Celisse, 2010) in the multivariate model including all traits. The difference in mean accuracy with or without the use of own performance was tested with a Student's t-test $(\mathrm{p}<0.001)$ for each trait.

\section{Results}

Basic statistics for all traits measured at slaughter are given in Table 1.

DNA parentage assignment success was $99.5 \%$. All parents were represented in the progeny. The observed number of full-sib families was 559 (93.1\% of the expected 600$)$. The average full-sib family size was $3.5 \pm 2.2$ and varied from 0 to 13 . Paternal (or maternal) half-sib family size varied from 8 to 45 (from 12 to 61 ).

Heritability at processing for the traits previously published (BW, Carc\%, Fil\%) for this experiment (Haffray et al., 2012a) and new traits (Fat\% and a*) is given in Table 2. The traits with the highest $\mathrm{h}^{2}$ were Fat\% $(0.55 \pm 0.04)$ and Carc\% $(0.49 \pm 0.04)$.

The mean accuracy of EBVs is given for combined family selection (using all data, including individual phenotype) or for sib selection (using LOO cross-validation - leaving out the individual phenotype) in Table 2. Accuracy was high for combined family selection and 
significantly higher (t-test; $\mathrm{p}<0.001)$ with multivariate models $(0.724-0.803)$ than with univariate models $(0.583-0.717)$, while both were significantly higher than the accuracy of sib-based selection with multivariate models $(0.587-0.627)$.

Non-genetic maternal effects were not significant (Haffray et al., 2012b), heritability was intermediate, and common environmental effects $\left(c^{2}\right)$ were supposed to be close to zero (all animals being reared in a common garden). Thus, the number of relatives of each individual was expected to determine $r_{A \hat{A}}$ (Van Vleck, 1993). Figure 1 shows the effect of the number of full-sibs or the number of full-sibs and half-sibs on the accuracy of EBV for Carc\%, as an example. When considering only the number of full-sibs of an individual (Figure 1a), there was a significant logarithmic regression between accuracy and the number of full-sibs $\left(r^{2}=\right.$ 0.52). However, some families with very few full-sibs ( 1 or 2$)$ had accuracies similar (0.64) to others with many more full-sibs $(>10)$. When also considering the number of half-sibs per family (Fig. 1b), the logarithmic regression was much improved $\left(r^{2}=0.73\right)$, with a total number of full and half-sibs for each candidate varying between 22 and 89 in the same dataset. Similar trends were observed for all the other traits.

\section{Discussion}

We estimated genetic parameters and breeding accuracies for individual and sib evaluated traits (growth and quality traits) in a mixed-family factorial mating design. Intermediate to high accuracy of EBVs was estimated even with a low number of full-sibs per family (3.3 on average) for combined family selection and sib-based selection.

Heritability estimates for BW, Fil\% and Carc\% (already published in Haffray et al., 2012a) and for new traits (Fat\% and $a^{*}$ ) were in the upper range of previous estimates already reported in rainbow trout (Kause et al., 2007). 
Intermediate to high accuracy of EBVs was estimated for all traits in combined family selection with a multivariate model when the traits were measured on all individuals $(0.630$ 0.817). These accuracy estimates were all above 0.5 , the maximum when using half-sibs in a univariate model, and some estimates were higher than 0.71 , the maximum when using fullsibs in a univariate model (Falconer and Mackay, 1996; Van Vleck, 1993). Logically, the lowest accuracy estimates were associated with the traits with the lowest heritability (Fil\% and $\left.\mathrm{a}^{*}\right)$ and the highest with the traits with the highest heritability (Car\% and Fat\%). These accuracy estimates were higher than those simulated in Solea solea (Blonk et al., 2010) for body weight $(0.558)$, with a much higher mean number of progenies per sire $(n=67.8)$ and per dam $(n=78.8)$. They were also higher than those simulated in Atlantic salmon $(0.558-$ 0.58) for a trait of intermediate theoretical heritability (0.40) based on 200 to 400 offspring per family for 200 independent full-sib families (Nielsen et al., 2011). They were still higher than those reported in a real breeding program for body weight (0.58) in Atlantic salmon (Tsai et al., 2015) with 712 individuals from 61 families, or those estimated in rainbow trout for fillet or gutted yield (0.54) based on 575 families phenotyped over two generations and a mean number of 4.65 individuals per family (Kause et al., 2007). Finally, these accuracy estimates were also much higher than those recently reported in rainbow trout for similar traits, such as the residual of fillet weight to body weight $(0.25)$ or fillet yield $(0.13)$ with five fish per family for 98 to 102 families produced over three generations (Gonzalez-Pena et al., 2016).

When only sib selection was considered (i.e., individual phenotype not used), accuracies decreased to intermediate levels $(0.587-0.627)$. They were in the same range as those simulated with univariate models in Atlantic salmon (0.56-0.58) in the only publication on sib-selection available that provides such estimates for sib selection (Lillehammer et al., 2013). 
Such intermediate to high breeding accuracy may be surprising considering our very low mean sib number per full-sib family (3.5-4), which was expected to lead to low accuracies. In mixed-family breeding programs using factorial designs, the number of full-sibs and half-sibs per candidate, not only the number of full-sibs, must be considered when discussing $r_{A \hat{A}}$ estimates. This hypothesis is in agreement with the positive effect of factorial mating structure on genetic gain proposed for genomic selection of sib-evaluated traits (Nirea et al., 2014). However, the number of full- and half-sibs was not sufficient to explain the residual variability of accuracy for a given number of sibs in Fig. 1b. This therefore implies variation in the number of cousins and half-cousins due to some parents being more or less related across and within factorial blocks. Finally, the combined use of a factorial mating design and BLUP estimations may thus compensate the very low number of full-sibs per family by also using additional information provided by the numerous genetic connections between factorials in the population.

Nevertheless, our results were obtained in a salmonid species with well-controlled reproduction and in which it is easy to produce factorial mating designs. Transfer to other situations (e.g., with natural spawning) or selection strategies, including genomics selection, would require further investigation to take into account the variations in biological or technical factors.

\section{Conclusions and perspectives for application}

This study is the first of its kind estimating genetic parameters, EBVs and $r_{A \hat{A}}$ of growth, processing traits and fillet quality in a mixed-family rearing design. The accuracy of EBVs was high, at least equivalent with those simulated in conventional (using separate families) breeding programs, and superior to those estimated from conventional breeding program data. This provides new insights for starting selection programs combining mass selection for 
growth and sib-based selection on quality traits. The results were obtained from a particular dataset and generalisation to other species or situations should be made with care.

The relative simplicity of this procedure and the high $r_{A \hat{A}}$ support the development of combined mass and sib-based selection programs without investment in specialized family rearing units, at least for species with limited market size or at an early stage in domestication. Preliminary results or simulations also highlighted the practical feasibility of using similar designs to integrate disease resistance from non-selected sibs (Chapuis et al., 2010; Norris et al., 2008).

\section{Competing interests}

The authors declare that they have no competing interests.

\section{Authors' contributions}

PH managed the project, estimated the heritabilities and $r_{A \hat{A}}$ and wrote the manuscript, FE estimated accuracy of EBVs and set up the LOO procedure, JB managed the data collection on $\mathrm{a}^{*}$ colour and Fat $\%, \mathrm{HC}$ supervised the estimation of genetic parameters and of EBV accuracy, MDN and MV supervised the genetic data treatment and the writing of the manuscript. All authors contributed to designing the study, interpreting the results, revising the manuscript and read and approved the final manuscript.

\section{Acknowledgements}

The authors are indebted to Cyrilles Deshayes and Marine Levadoux from the French Interprofessional Committee for Aquaculture Products (CIPA) for their support and management of the project and the staff of INRA, IFREMER and French breeding companies who are members of SYSAAF (Aqualande, Viviers de France, Font Rome Pisciculture and 
Les Fils de Charles Murgat) for their participation in the fish rearing and data collection. This work was supported by the French National Interprofessional Office for Marine and Aquaculture Products (OFIMER) (convention no. 083/08/C) and by the European Union through the European Fisheries Fund (EFF, convention no. 30900/2009). 
Table 1: Phenotypic performance at slaughter.

\begin{tabular}{|l|c|c|c|}
\hline \multicolumn{1}{|c|}{ Trait } & Mean \pm SD & CV\% & N \\
\hline Body weight in g (BW) & $1639 \pm 351$ & 21.4 & 1962 \\
\hline Carcass yield in \% (Carc\%) & $87.7 \pm 1.4$ & 1.6 & 1948 \\
\hline Fillet yield in \% (Fil\%) & $66.8 \pm 2.0$ & 3.0 & 1925 \\
\hline Fat \% & $8.9 \pm 1.1$ & 12.4 & 1876 \\
\hline Red saturation (a*) & $9.1 \pm 1.0$ & 11.0 & 1910 \\
\hline
\end{tabular}

Mean, standard deviation (SD), coefficient of variation $(\mathrm{CV} \%)$ and number of fish $(\mathrm{N})$ considered in the genetic analysis. 
Table 2: Heritability and accuracy of EBVs for combined family selection or sib-based selection with multivariate or univariate models

\begin{tabular}{|c|c|c|c|c|}
\hline Trait & Heritability & $\begin{array}{c}\text { Combined } \\
\text { family } \\
\text { selection with } \\
\text { univariate } \\
\text { model }\end{array}$ & $\begin{array}{c}\text { Combined family } \\
\text { selection with } \\
\text { multivariate } \\
\text { model }\end{array}$ & $\begin{array}{c}\text { Sib selection with } \\
\text { multivariate } \\
\text { model }\end{array}$ \\
\hline Body weight (BW) & $0.37(0.04)$ & $0.717 \pm 0.009$ & $0.730 \pm 0.015$ & $0.598 \pm 0.068$ \\
\hline Carcass yield (Carc\%) & $0.49(0.06)$ & $0.626 \pm 0.015$ & $0.776 \pm 0.017$ & $0.618 \pm 0.069$ \\
\hline Fillet yield (Fil\%) & $0.31(0.05)$ & $0.583 \pm 0.020$ & $0.724 \pm 0.016$ & $0.587 \pm 0.067$ \\
\hline Fat content of the fillet (Fat\%) & $0.55(0.06)$ & $0.632 \pm 0.015$ & $0.803 \pm 0.037$ & $0.627 \pm 0.070$ \\
\hline Red colour saturation of the fillet (a*) & $0.30(0.05)$ & $0.587 \pm 0.020$ & $0.736 \pm 0.015$ & $0.592 \pm 0.067$ \\
\hline
\end{tabular}

Heritability and mean accuracy ( \pm standard deviation) of EBV estimated for combined family selection with a univariate or multivariate model or sib-based selection with a multivariate model for growth $(\mathrm{BW})$, processing traits (Carc\% and Fil\%) or fillet quality traits (Fat\% and $\left.a^{*}\right)$ 
Figure 1:

Effect of the number of full-sibs or the sum of full-sibs and half-sibs on EBV accuracy of the candidates of the CAND group for Carc\% using sib information from the REF group.

Legend of Figure 1: Familial EBV accuracy as a function of the number of 1a) sib number per family; 1b) full- and half-sib number per family. 
References

Arlot, S., and Celisse, A. (2010). A survey of cross-validation procedures for model selection. Statist. Surv. 4, 40-79.

Blonk, R. J. W., Komen, J., Tenghe, A., Kamstra, A., and van Arendonk, J. A. M. (2010). Heritability of shape in common sole, Solea solea, estimated from image analysis data. Aquaculture 307, 6-11.

Chapuis, H., Vandeputte, M., Dupont-Nivet, M., Haffray, P., and Quillet, E. (2010). Selection for an improved disease resistance using factorial mating designs and molecular based pedigrees in fish: a simulation study. Proceedings of the 9th World Congress on Genetics Applied to Livestock Production, Leipzig, Germany, CD Rom Paper, $N^{\circ} 0210$.

CIE (1976). Official recommendations on uniform colour space, colour difference equations and metric colour terms. Suppl. $N^{\circ} 2$ to CIE publication $N^{\circ} 15$.

Douirin, C., Haffray, P., Vallet, J. L., and Fauconneau, B. (1998). Determination of the lipid content of rainbow trout (Oncorhynchus mykiss) filets with the Torry Fish Fat Meter (R). Sciences Des Aliments 18, 527-535.

Dupont-Nivet, M., Vandeputte, M., Haffray, P., and Chevassus, B. (2006). Effect of different mating designs on inbreeding, genetic variance and response to selection when applying individual selection in fish breeding programs. Aquaculture 252, 161-170.

Falconer, D. S., and Mackay, T. F. C. (1996). "Introduction to quantitative genetics," Harlow: Pearson Education Limited.

Gjedrem, T., Robinson, N., and Rye, M. (2012). The importance of selective breeding in aquaculture to meet future demands for animal protein: A review. Aquaculture 350, 117-129.

Gonzalez-Pena, D., Gao, G. T., Baranski, M., Moen, T., Cleveland, B. M., Kenney, P. B., Vallejo, R. L., Palti, Y., and Leeds, T. D. (2016). Genome-Wide Association Study for Identifying Loci that Affect Fillet Yield, Carcass, and Body Weight Traits in Rainbow Trout (Oncorhynchus mykiss). Frontiers in Genetics 7.

Haffray, P., Bugeon, J., Pincent, C., Chapuis, H., Mazeiraud, E., Rossignol, M. N., Chatain, B., Vandeputte, M., and Dupont-Nivet, M. (2012a). Negative genetic correlations between production traits and head or bony tissues in large all-female rainbow trout (Oncorhynchus mykiss). Aquaculture 368, 145-152.

Haffray, P., Bugeon, J., Rivard, Q., Quittet, B., Puyo, S., Allamelou, J. M., Vandeputte, M., and Dupont-Nivet, M. (2013). Genetic parameters of in-vivo prediction of carcass, head and fillet yields by internal ultrasound and 2D external imagery in large rainbow trout (Oncorhynchus mykiss). Aquaculture 410, 236-244.

Haffray, P., Vandeputte, M., Petit, V., Pincent, C., Chatain, B., Chapuis, H., Meriaux, J. C., Coudurier, B., Quillet, E., and Dupont-Nivet, M. (2012b). Minimizing maternal effect in salmonid families mixed since eyed stages and a posteriori DNA-pedigreed. Livestock Science 150, 170-178.

Henderson, C. R. (1984). "Applications of linear models in animal breeding.," Third Edition by L. R. Schaeffer/Ed.

Kause, A., Paananen, T., Ritola, O., and Koskinen, H. (2007). Direct and indirect selection of visceral lipid weight, fillet weight, and fillet percentage in a rainbow trout breeding program. Journal of Animal Science 85, 3218-3227.

Koch, R. M., Gregory, K. E., Chambers, D., and Swiger, L. A. (1963). EFFICIENCY OF FEED USE IN BEEF CATTLE. Journal of Animal Science 22, 486-\&. 
Lillehammer, M., Meuwissen, T. H. E., and Sonesson, A. K. (2013). A low-marker density implementation of genomic selection in aquaculture using within-family genomic breeding values. Genetics Selection Evolution 45.

Misztal, I., Tsuruta, S., Strabel, T., Auvray, B., Druet, T., and Lee, D. (2002). BLUPF90 and related programs (BGF90). Proceedings of the 7th world congress on genetics applied to livestock production, Montpellier, France, communication No. 28-27, 28:21-22.

Nielsen, H. M., Sonesson, A. K., and Meuwissen, T. H. E. (2011). Optimum contribution selection using traditional best linear unbiased prediction and genomic breeding values in aquaculture breeding schemes. Journal of Animal Science 89, 630-638.

Nirea, K., Sonesson, A. K., Lillehammer, M., and Meuwissen, T. ( 2014). Effect of family structure and size on genomic selection accuracy in aquaculture breeding. Proceedings, 10th World Congress of Genetics Applied to Livestock Production.

Norris, A., Foyle, L., and Ratcliff, J. (2008). Heritability of mortality in response to a natural pancreas disease (SPDV) challenge in Atlantic salmon, Salmo salar L., post-smolts on a West of Ireland sea site. Journal of Fish Diseases 31, 913-920.

Sorensen, A. C., Berg, P., and Woolliams, J. A. (2005). The advantage of factorial mating under selection is uncovered by deterministically predicted rates of inbreeding. Genetics Selection Evolution 37, 57-81.

Tsai, H. Y., Hamilton, A., Tinch, A. E., Guy, D. R., Gharbi, K., Stear, M. J., Matika, O., Bishop, S. C., and Houston, R. D. (2015). Genome wide association and genomic prediction for growth traits in juvenile farmed Atlantic salmon using a high density SNP array. Bmc Genomics 16.

Van Vleck, D. L. (1993). "Selection index and introduction to mixed model methods."

Vandeputte, M., and Haffray, P. (2014). Parentage assignment with genomic markers: a major advance for understanding and exploiting genetic variation of quantitative traits in farmed aquatic animals. Frontiers in Genetics 5. 


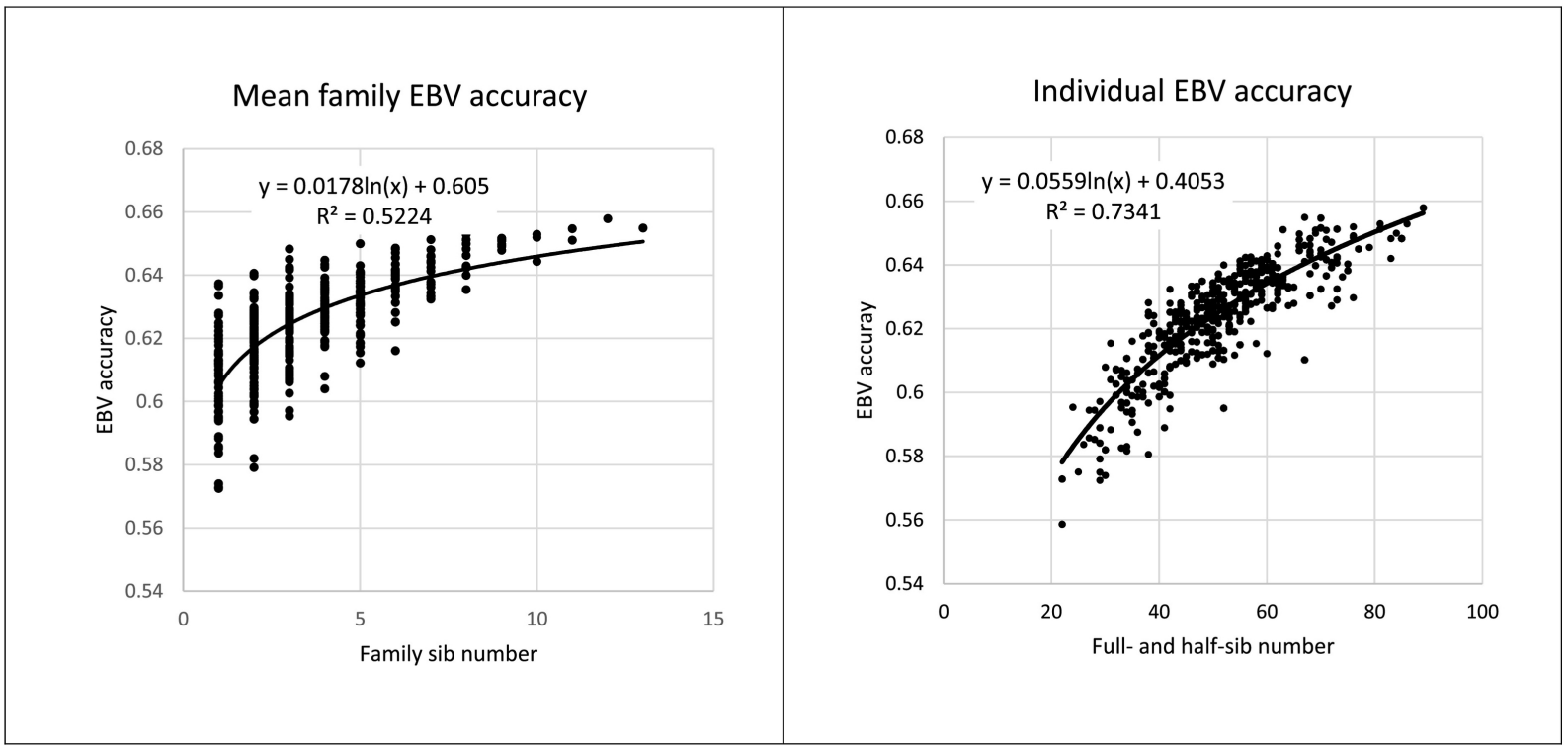

Figure 1 